Case Report

\title{
Xanthogranulomatous Inflammation of the Female Genital Tract: Report of Three Cases
}

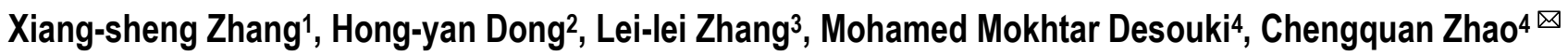 \\ 1. Department of Pathology, Binzhou Medical College, Binzhou 256603, China; \\ 2. Department of Pathology, People Hospital of Linyi City, Shandong Provine, China; \\ 3. Department of gynecology and obstetrics, Binzhou Medical College, Binzhou 256603, China; \\ 4. Department of Pathology, Magee-Womens Hospital of University of Pittsburgh Medical Center, Pittsburgh, PA 15213, \\ USA.
}

$\triangle$ Corresponding author: Chengquan Zhao, MD., Magee Womens Hospital, 300 Halket Street, Pittsburgh, PA 15213. Office Phone: (412) 641-6678 Office Fax: (412) 641-1675 Email Address: czhao@mail.magee.edu.

(C) Ivyspring International Publisher. This is an open-access article distributed under the terms of the Creative Commons License (http://creativecommons.org/ licenses/by-nc-nd/3.0/). Reproduction is permitted for personal, noncommercial use, provided that the article is in whole, unmodified, and properly cited.

Received: 2011.12.07; Accepted: 2012.01.25; Published: 2012.02.23

\begin{abstract}
Purpose and Methods: This is a series of three cases diagnosed with xanthogranulomatous inflammation of the female genital with emphasis on the etiology, clinical-pathologic features and biological behavior. Clinical, pathologic, radiologic and follow up data are reported.

Results: The three cases of Xanthogranulomatous inflammation of the female genital tract are the followings: I) one case affecting the endometrium, 2) one case affecting the fallopian tube, and 3) one case confined to the ovary. The patient's age was 37, 22 and 62 year-old, respectively. Histologic examination revealed extensive infiltration of foamy histiocytes admixed with variable amount of inflammatory cells. The later include plasma cells, lymphocytes, and occasional multinucleated giant cells. Immunohistochemistry showed positive staining for CD68, a histiocytic marker, in foamy histiocytes, CD3, a T cell marker, and CD20, a B cell marker, in the background lymphocytes. The plasma cells were polyclonal with expression of both $\mathrm{K}$ and $\lambda$ light chains.

Conclusion: Xanthogranulomatous inflammation of the female genital tract is an unusual lesion, and clinically forms mass- like lesion in the pelvic cavity that invades the surrounding tissues, which may mimic the tumor clinically and by imaging.
\end{abstract}

Key words: xanthogranulomatous inflammation; endometritis; salpingitis; oophoritis.

\section{Introduction}

Xanthogranulomatous inflammation is a special form of chronic inflammation that is destructive to normal tissue of affected organs. This is an uncommon process mostly affecting the kidney. However, other organs in which xanthogranulomatous inflammation has been reported are the gallbladder, stomach, anorectal area, bone, urinary bladder, testis, epididymis, vagina and endometrium. Xanthogranulomatous inflammation affecting the female genital tract is rare. It is characterized by a massive infiltration of the tissues by lipid-laden histiocytes admixedwith lymphocytes, plasma cells and polymorphonuclear leucocytes. There is no minimal amount requirement for the histiocytes to make this diagnosis.

Clinically and radiologically, Xanthogranulomatous inflammation is mimicking tumor of the ovary and fallopian tube. Kunakemakorn was the first to report inflammatory pseudotumor in the pelvis in 
1976 [1]. To date, only 13 cases of xanthogranulomatous inflammation in the ovary and fallopian tubes [1-10], and 15 cases in the endometrium have been reported in the English literature [11-15]. In this paper we report three cases of xanthogranulomatous inflammation in the female genital tract with review of the literature on this subject.

\section{Case reports}

\section{Case I}

Clinical and radiologic findings

A 32-year-old woman originally presented to her gynecologist with lower abdominal pain, colporrhagia and fever. The patient had a poor appetite and anorexia with a temperature of $38^{\circ} \mathrm{C}$. Physical examination revealed palpable liver $2 \mathrm{~cm}$ under the costal margin at the mid-clavicular line. There was vague tenderness in the costophrenic angle on percussion. A left ovarian mass was found on gynecological examination.

Computer tomography (CAT) scan revealed a well-defined solid mass with different signal intensity centrally, interpreted as ovarian carcinoma. Hysterectomy and bilateral salpingo-oophorectomy surgical intervention was performed.

Histo-pathological findings

Gross pathology: The uterus measured $7 \times 4 \times 3$ $\mathrm{cm}$ with smooth endometrium, the outer serosal lin- ing was adherent to the broad ligament and ovaries. The left ovary had a solid mass measured $4 \times 3 \times 3 \mathrm{~cm}$ with rugged outer surface. On serial sectioning, there were grayish-white and necrotic mass with focal hemorrhage and variable-thickness pseudo-capsule.

Microscopic examination: All specimens were fixed in $10 \%$ formalin, dehydrated in descending grades of alcohol, sections were paraffin embedded and processed for histologic and immunohistochemical stains. The pathological changes were composed of mixed inflammatory cells and focal foamy histiocytes. The inflammatory cells were mainly lymphocytes admixed with plasma cells, small number of neutrophils and eosinophils (Figure 1). The foamy histiocytes were focally scattered, with abundant cytoplasm, small lipid vacuoles and hypochromatic nuclei (Figure 2). Multinucleated giant cells were not observed. In the stroma, fibroplasia with fibrosis, vascular proliferation with sclerosis in arterioles, hyperplasia and swelling in blood capillary endothelial lining were observed. The fibrous pseudocapsule was infiltrated with chronic inflammatory cells. Periodic acid-schiff (PAS) and acid fast stains were negative. The subsequent immunohistochemical stains demonstrated positive CD20, CD5, CD79a, $\mathrm{k}$ and $\lambda$. The pathological diagnosis rendered as xanthogranulomatous oophoritis.

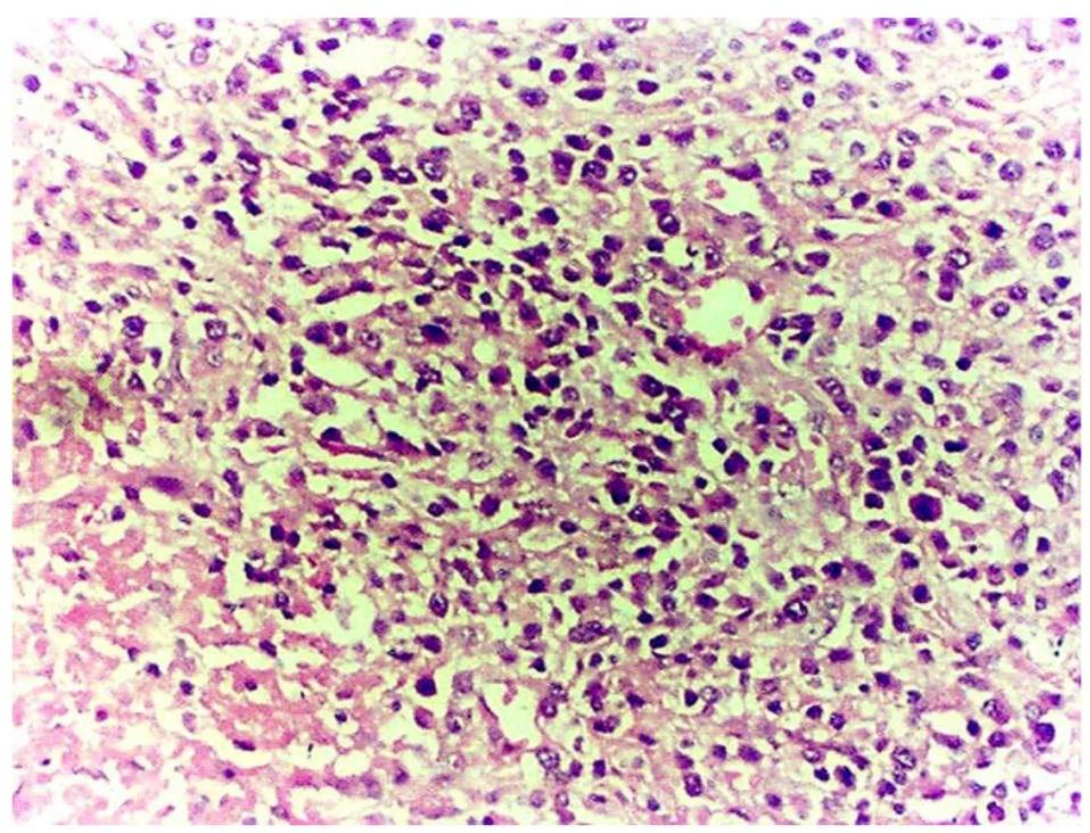

Figure I. Effaced ovarian structure with dense chronic inflammatory cellular infiltrate (case I). 


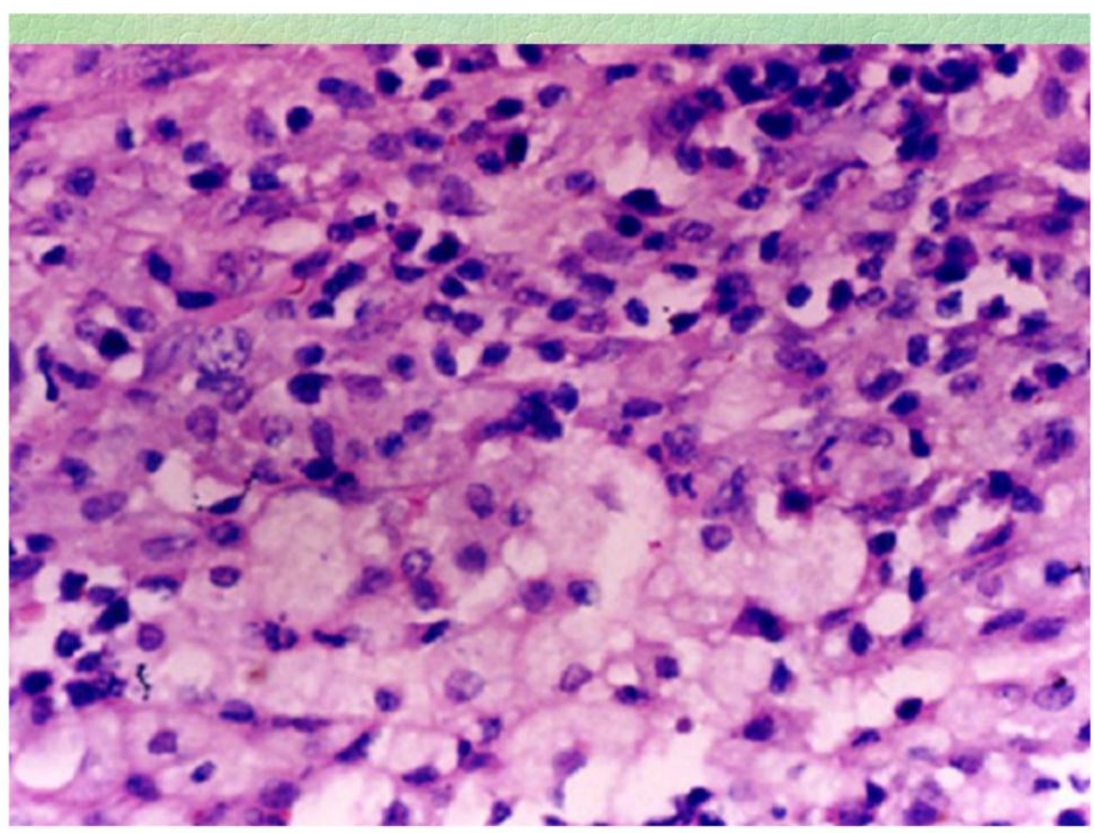

Figure 2. Foamy histiocytes in higher power view (case I).

\section{Case 2}

Clinical and radiologic findings

Twenty two-year-old woman presented with lower abdominal discomfort, fever, excessive leucorrhea and anorexia on admission. Physical examination revealed tenderness in the lower abdominal region with deep palpation and palpable liver under the costal margin in the midclavicular line. Gynecological examination revealed erosion of the cervix with tenderness, and vague-defined mass measuring $6 \times 4 \times 4$ $\mathrm{cm}$ in the left adnexal region confirmed by ultrasonographic examination as a solid mass with septation measuring $6.3 \times 4.7 \times 3.6 \mathrm{~cm}$. Follow-up ultrasonography, performed after two weeks of antibiotics treatment, demonstrated decrease in the size to $3.5 \times$ $3.2 \times 2.4$. However, the left ovary increased in size to $7.9 \mathrm{~cm} \times 4.3 \mathrm{~cm} \times 2.7 \mathrm{~cm}$.

Subsequently, a left salpingo-oophorectomy was performed. During the operation, there was adhering zone on the serosal surface. The left fallopian tube measured $3.0 \mathrm{~cm}$ in diameter and $5 . \mathrm{cm}$ in length with small amount of effusion on the serosal surface with clogged fimbriated end. The left ovary was visibly edematous with a $3.0 \mathrm{~cm}$ cyst on cross section. The right fallopian tube was similar to the left with a nodule that measure $2.5 \mathrm{~cm}$. The clinical diagnosis was bilateral salpingitis and cystic mass of the left ovary.

\section{Histo-pathological findings}

Gross pathology: The left fallopian tube was 4.6 $\mathrm{cm}$ in length, $1.9 \mathrm{~cm}$ in diameter, and $0.3 \mathrm{~cm}$ in thickness. There was small amount of exudate on the external surface and serous fluid in the stenotic lumen.

Microscopic examination: The visibly-decreasing fallopian tube plicae were distended and covered by low-columnar glandular epithelium. There were infiltration by xanthoma cells admixed with inflammatory cells such as lymphocytes, plasma cells and neutrophils (Figures 3-4), accompanied with fibroplasia and vascular proliferation in the stroma. The xanthoma cells present either focally or in sheets, with no giant cells identified. A cyst-wall-like structure identified on the left ovary which measured $4 \times 3$ $\times 1.3 \mathrm{~cm}$. The subsequent immunohistochemical stains showed CD68 positivity in the xanthoma cells, $\mathrm{CD} 3, \mathrm{CD} 20, \mathrm{~K}$ and $\lambda$ were positive in the lymphocytes and pan cytokeratin was positive in the glandular epithelium. Pathological diagnosis rendered left xanthogranulomatous salpingitis and follicular cyst of left ovary.

\section{Case 3}

\section{Clinical and radiologic findings}

A post-menopausal, 62-year-old woman admitted for paroxysmal vague pain in the lower abdominal region, intermittently low-grade fever and lassitude. Physical examination finding was inconspicuous tenderness in lower abdomen with deep palpation. Gynecological examination revealed erosive cervix with redness. On ultrasonographic examination, the uterus measured $5.7 \times 5.1 \times 4.2 \mathrm{~cm}$ with 
uneven endometrium. A transvaginal dilatation and curettage was performed.

\section{Histo-pathological findings}

Gross pathology: Multiple fragments of endometrium measuring $2.4 \times 1.3 \times 1.0 \mathrm{~cm}$ in aggregate

Microscopic examination: The glandular architecture of the endometrium was preserved, and the lining epithelial cells were focally crowded in considerable fields. The stroma was infiltrated by focal or sheets of foam cells admixed with lymphocytes, plasma cells and occasional neutrophils (Figure 5). These foam cells had light staining, comparatively small nuclei surrounded by abundant cytoplasm containing lipid vacuoles. Immunohistochemical stains demonstrated CD68 positivity in the foam cells, CD20 and CD3 were positive in the lymphocytes. The endometrial epithelium was not reactive to ER and PR. The pathological diagnosis rendered xanthogranulomatous endometritis.

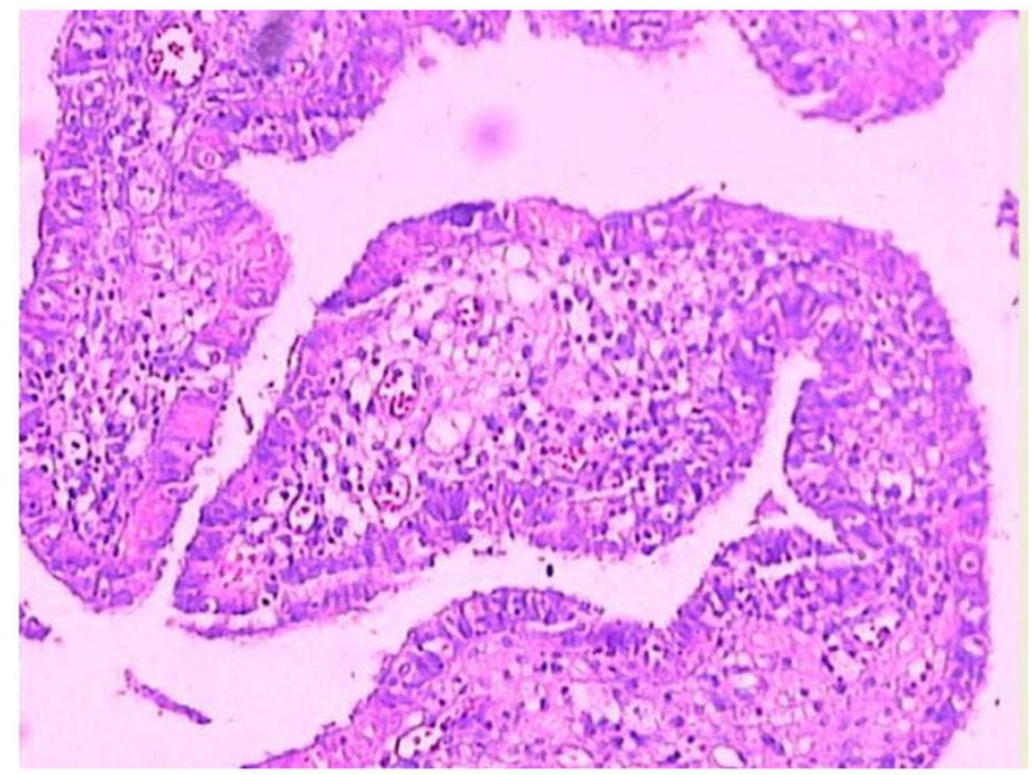

Figure 3. Infiltration of the lamina propria of the fallopian tube plicae with foamy histiocytes and chronic inflammatory cells (case 2).

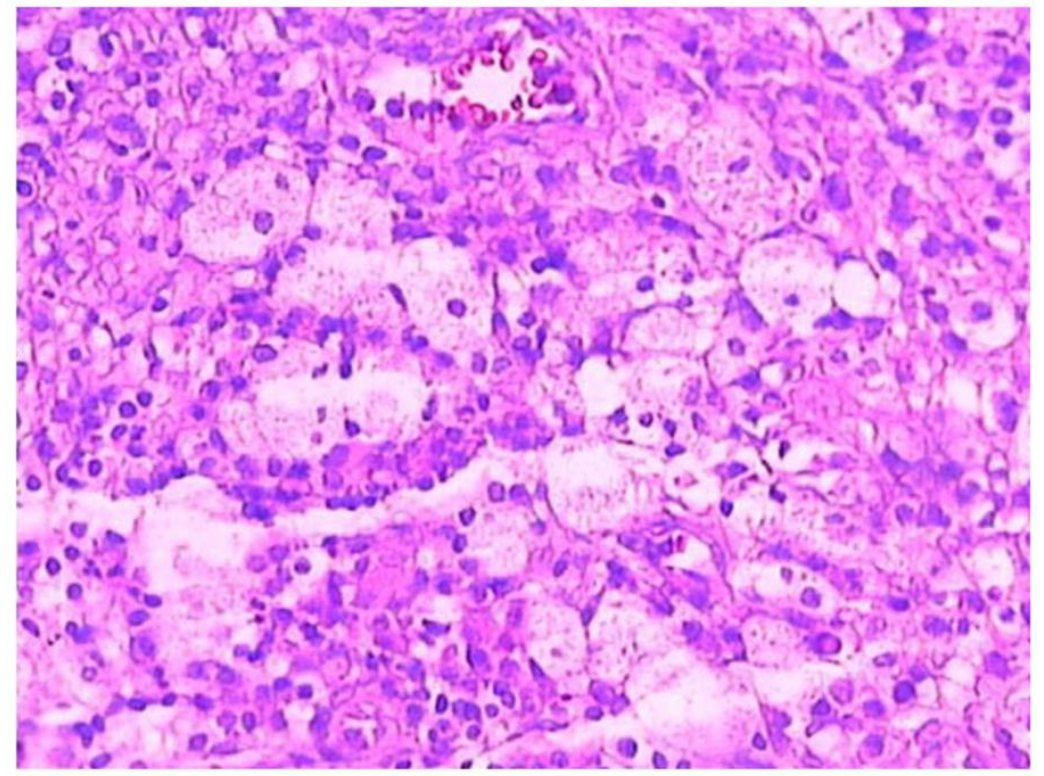

Figure 4. Clusters of foam cells in the lamina propria of the fallopian tube in high power view (case 2). 


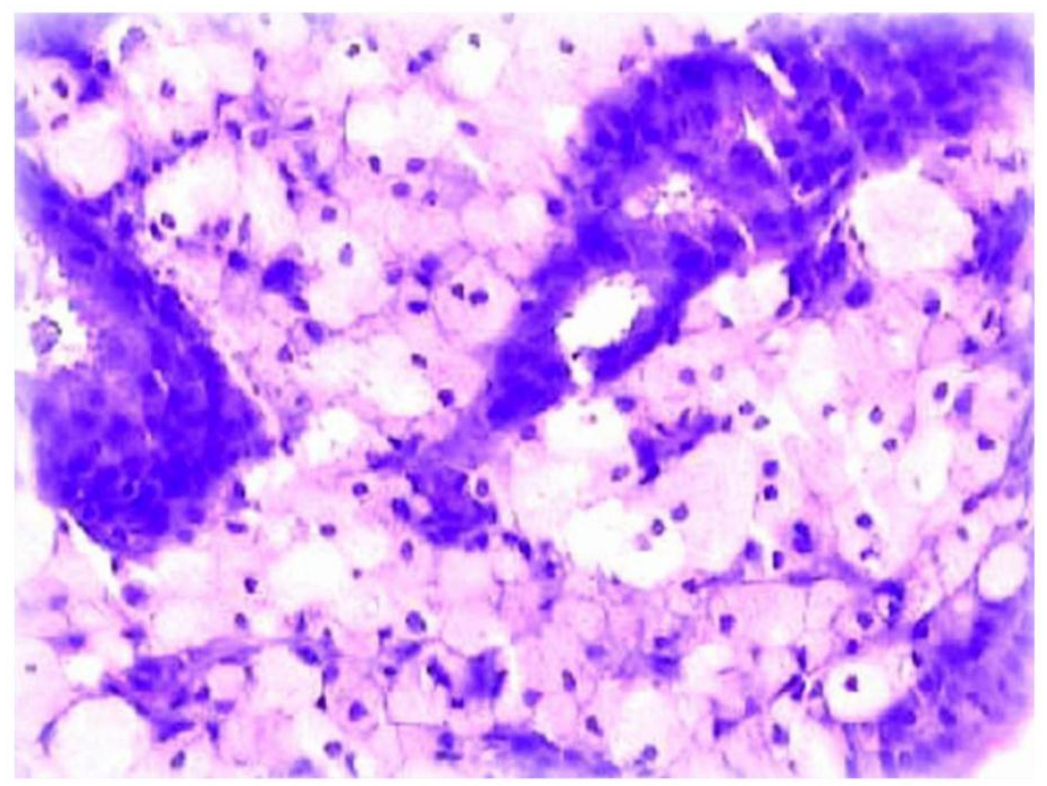

Figure 5. Disintegration of the endometrial glands accompanied by infiltration of abundant foam cells.

\section{Discussion}

Xanthogranulomatous inflammation in the female genital tract has different terminology such as xathogranulomatous salpingitis and ovarian fibroxanthoma [8, 9]. The affected organs suffer disorganization and infiltration with focal or sheets of foam cells admixed with chronic inflammatory cells such as lymphocytes, plasma cells, occasional neutrophils with or without multinucleated or Touton giant cells. Xanthogranulomatous inflammation occurring in female genital tract affects endometrium, fallopian tubes or ovaries focally or entirely, which clinically forms mass- like lesion in the pelvic cavity and invades the surrounding tissues.

Only 13 related cases of Xanthogranulomatous inflammation involving fallopian tube or ovary have been described so far. According to the reported and our cases, the clinical-pathological features are summarized as following [1-10]: 1) Xanthogranulomatous inflammation in female genital tract mainly affects one side; 7 cases involving unilateral ovary, 5 cases involving unilateral fallopian tube, 1 case with bilateral fallopian tubes, 2 cases with ovaries and fallopian tubes simultaneously. The ovaries, fallopian tubes and broad ligament adhere to the uterine surface on different degree. 2) Patients usually present with history of pelvic inflammatory disease lasting from months to years. Antibiotic therapy is ineffective, or probably nonsensitive antibiotics were used. 3) The average age is 38.5 years (range 23-72), the clinical presentations include anemia, anorexia,, fever, irreg- ular colporrhagia, and pain in the hypogastrium and/or upper abdomen. Gynecological examination reveals adnexal mass with tenderness. 4) Laboratory tests showed elevated ESR and white blood cell count. 5) ultrasound and CAT scans demonstrate a well-defined solid mass.

The clinical manifestation, imaging detection and macroscopic observation of xanthogranulomatous oophoritis are subject to be confused with ovarian malignancy. Seven reported cases as well as ours were all misdiagnosed as ovarian cancers. The mass is unilateral, measuring 3-7 $\mathrm{cm}$ in maximum dimension. It has comparatively clear borders with no intact capsule, which adheres to fallopian tubes, broad ligament and uterine surface. The cross section is solid, fragile, frequently accompanied with hemorrhage, necrosis and cystic degeneration. Morphologically, the normal ovarian structure is destroyed and replaced by chronic inflammatory cell infiltrate admixed with focal or sheets of foam cells, fibroplasia and vascular proliferation.

Xanthogranulomatous oophoritis is often misdiagnosed by pathologists if they don't keep this entity in mind. This may be due to the rarity of the condition. If the lesion is mainly focal scattered lymphocytes, it may be misdiagnosed as secondary lymphoma or leukemia. If the lymphocytes are scattering diffusely and foam cells are seldom, a diagnosis of malignant small cell tumor with stromal luteinization may be rendered. If there are small amount of obvious fibrosis and foam cells, a diagnosis of sclerosing stromal tumor may be made. However, the right di- 
agnosis is possible to be made as long as pathologists elevate vigilance and master the pathological features. Immunohistiochemical stains are helpful in establishing the diagnosis, including CD68 (foam cells positive), CD3 (T lymphocyte marker), CD20 (B lymphocytes marker), $\mathrm{K}$ and $\lambda$ (both positive in polyclonal B lymphocytes).

Xanthogranulomatous salpingitis mainly involves unilateral fallopian tubes, occasionally occurs bilaterally. It is clinically often accompanied with inflammation in vagina, cervix and endometrium. The visibly thickened fallopian tube is variable in length, with an appearance of mass lesion. There is exudation on the serosal surface and the fimbriated end of fallopian tube is usually obliterated. Morphologically, the fallopian tube structure remains intact. The enlarging plicae is covered by degenerated or necrotic epithelium and infiltrated by a large number of foam cells admixed with lymphocytes, plasma cells and seldom neutrophils in the lamina propria. Multinucleated giant cells can also be seen. A case of xanthogranulomatous salpingitis accompanied with endometriosis have been reported by Idrees et al[10]. This condition should be differentiated from metastatic well-differentiated adenocarcinoma.

Xanthogranulomatous endometritis was also named as histiocytic endometritis, pseudoxanthomatous endometritis beside other names. The age at onset ranges from 59-88 years, with an average age of 72 years. The clinical presenting symptoms are irregular coporrhagia, excessive leucorrhea, cervical stenosis or pyometra. There are two groups of xanthogranulomatous endometritis; one is pure xanthogranulomatous inflammation, the other is xanthogranulomatous endometritis accompanied with endometrial cancer. Among the 15 reported cases; 9 accompanied with endometrial cancer. The histological changes are similar to those in fallopian tubes. Nevertheless, the affected endometrial glands may disappear completely and disintegrate, occasionally be necrotic. There is usually exudation covering the endometrial serosal surface. The concomitant cancer mainly is endometrioid carcinoma. The prognosis varies in reports; some cases recovered spontaneously after antibiotic therapy, whereas some other cases were unresponsive to antibiotic treatment. However, it is quite often to seen large amount of histiocytes associated with cancer, especially after chemotherapy.

Xanthogranulomatous inflammation of female genital tract is an inflammatory disease, the etiologic factor and pathogenicity of which are not clear yet. Many theories have been proposed, such as theory of infection, endometriosis, intrauterine contraceptive device, inborn lipid metabolism of macrophage and drugs (antibiotics) etc. The perceived theory is the theory of infection, which is supported by history of clinical infection and bacteria such as Escherichia coli, Bacillus, salmonella beside others $[2,3,5]$ which may be grown from the affected tissue by culture. However, the large amount of foam cells containing lipids is hard to be elucidated by the theory of infection exclusively. Analyzed from the reported cases, the emergence of foam cells may be relevant to the following factors. The first one is inefficient or inappropriate antibiotics applied in the early phase of infection which didn't control the multiplication of bacteria. Macrophages phagocytize the lipid and change into foam cells. The second one is lipid metabolic disorder induces hyperlipidemia, and the foam cells are formed when the lipid deposited in the affected region is phagocytosed by phagocytes. The third one is the application of intra-uterine contraceptive device or drugs, the later contains lipoid substance. Wather ${ }^{[16]}$ presumed malakoplakia and xanthogranulomatous inflammation were identical chronic inflammatory disease. In malakoplakia, the cytoplasmic concentric calcific bodies (Michaelis-Gutmann bodies) are phagolysosome of phagocytes which calcifies after phagocytes phagocytize E. coli but fail to digest it. Malakoplakia occurs mainly in the urinary system, but xanthogranulomatous inflammation occurs mainly in the genital system.

\section{Conflict of Interest}

The authors have declared that no conflict of interest exists.

\section{References}

1. Kunakemakorn $\mathrm{P}$, Ontai H. Pelvic inflammatory pseudotumor: A case report. Am J Obstet Gynecol ,1976;26: 286-287.

2. Shalev E, Zuckerman H, Rizescu I. Pelvic inflammatory pseudotumor (Xanthogranuloma). Acta Obstet Gynecol Scand, 1982; 61: 285-286.

3. Pace EH, Voet RL, Melancon JT, et al. Xanthogranulomatous oophoritis: an inflammatory pseudotumor of the ovary. Int J gynecol Pathol,1984;3: 398-402.

4. Davis M, Whitley ME, Haque AK, et al. Xanthogranulomatous abscess of a mullerian duct remnant: rare lesion of the rectum and anus. Dis Colon Rectum,1986;29: 755-759.

5. Ladetoged C, Loretzen M. Xanthogranulomatous inflammation of th female genital tract. Histopathology ,1988;13: 541-551.

6. Franco V, lorena AM,Guarneri G, et al. Xanthogranulomatous salpingitis:case report and review of the literature. Acta Eur Fertil, 1990; 21: 197-199.

7. Bates AW, Fegan AW, Baithun SL. Xanthogranulomatous cystitis associated with malignant neoplasms of the bladder. Histopathology, 1998; 33: 212-215.

8. Gray Y, Libbey NP. Xanthogranulomatous salpingitis and oophoritis:A case report and review of the literature. Arch Pathol Lab Med, 2001; 125: 260-263.

9. Furuya M, et al. Pseudoxanthomatous and Xanthogranulomatous Salpingitis of the Fallopian Tube: A Report of Four Cases 
and a Literature Review. Interna J Gynecol Patho, 2001; 21: 56-59,

10. Idrees M, Zakashansky K, Kalir T. Xanthogranulomatous salpingitis associated with fallopian tube mucosal endometriosis: a clue to the pathogenesis. An Diag Pathol 2007; 11: 117-121.

11. Barua R, Krikland JA, Petrucco OM. Xanthogranulomatous endometritis: case report. Pathology, 1978;10: 161-164.

12. Pounder DJ, Iyer PV. Xanthogranulomatous endometritis associated with endometrial carcinoma. Arch Pathol Lab Med. 1985;109: 73-75.

13. Russack V, Lammers RJ. Xanthogranulomatous endometritis: Report of six cases and a proposed mechanism of development. Arch Pathol Lab Med. 1990;114: 929-932.

14. Noack F, Briese J, Stellmacher FD, et al. Lethal outcome in xanthogranulomatous endometritis. APMIS. 2006; 114: 386-388.

15. Dogan-Ekici I, Usubutun A, Kucukali T, et al. Xanthogranulomatous Endometritis: A Challenging Imitator of Endometrial Carcinoma. Infect Dis Obstet Gynecol, 2007; 2007:34763.

16. Walther M, Glenn JF, Vellios F. Xanthogranulomatous cystitis. J Urol, 1985;134: 745-746. 